\title{
Numerical Investigation of Turbulence Models for a Superlaminar Journal Bearing
}

\author{
Aoshuang Ding $\mathbb{D}^{D}$, Xiaodong Ren, Xuesong Li ${ }^{\mathbb{D}}$, and Chunwei Gu \\ Department of Thermal Engineering, Tsinghua University, Beijing 100084, China \\ Correspondence should be addressed to Xuesong Li; xs-li@mail.tsinghua.edu.cn
}

Received 11 July 2017; Accepted 4 December 2017; Published 3 January 2018

Academic Editor: Michel Fillon

Copyright (C) 2018 Aoshuang Ding et al. This is an open access article distributed under the Creative Commons Attribution License, which permits unrestricted use, distribution, and reproduction in any medium, provided the original work is properly cited.

\begin{abstract}
With rotating machineries working at high speeds, oil flow in bearings becomes superlaminar. Under superlaminar conditions, flow exhibits between laminar and fully developed turbulence. In this study, superlaminar oil flow in an oil-lubricated tilting-pad journal bearing is analyzed through computational fluid dynamics (CFD). A three-dimensional bearing model is established. CFD results from the laminar model and 14 turbulence models are compared with experimental findings. The laminar simulation results of padside pressure are inconsistent with the experimental data. Thus, the turbulence effects on superlaminar flow should be considered. The simulated temperature and pressure distributions from the classical fully developed turbulence models cannot correctly fit the experimental data. As such, turbulence models should be corrected for superlaminar flow. However, several corrections, such as transition correction, are unsuitable. Among all the flow models, the SST model with low-Re correction exhibits the best pressure distribution and turbulence viscosity ratio. Velocity profile analysis confirms that a buffer layer plays an important role in the superlaminar boundary layer. Classical fully developed turbulence models cannot accurately predict the buffer layer, but this problem can be resolved by initiating an appropriate low-Re correction. Therefore, the SST model with low-Re correction yields suitable results for superlaminar flows in bearings.
\end{abstract}

\section{Introduction}

Lubricant flow in bearings has been considered laminar and lubricant cavitation has been extensively investigated. Tensile stress has also been explored to determine the mechanisms of vaporous and gaseous cavitation [1-3]. Two cavitation mechanisms have been compared under static and dynamic loads to identify the dominant mechanism [4-6]. A new model based on air solubility has also been proposed for gaseous cavitation $[7,8]$ and improved by considering nonequilibrium dissolution for oil film in bearings [9]. A tapered land thrust bearing has also been utilized to verify the cavitation effect $[10,11]$. The relationship between temperature and cavitation has been evaluated [12-15].

With rotating machineries working at high rotating speeds on a large scale, the performances of journal bearings weaken as friction loss increases and the lubricant flow regime of journal bearings changes from laminar to superlaminar or turbulent. Considering the significantly high Reynolds number of a fully developed turbulent flow, oil flow regime in high-speed journal bearings is superlaminar [16]. Thus, superlaminar journal bearings should be accurately predicted to improve the performance of these bearings.

Superlaminar oil films in bearings have been explored by considering turbulence effect. The turbulence effect on bearing lubricant flows was initially examined on the basis of mixing length theory. Compared with the laminar simulation results and experimental data, turbulence simulation results confirm that turbulence effect should be considered for bearing lubricant flows [17, 18]. Ng and Pan [19] further investigated the theories based on law of wall and mixing length theory. Considering the verified theoretical results based on law of wall, Hirs [20] proposed a bulk-flow theory for bearing lubricant simulation. Suganami and Szeri [21] examined the thermal effect and confirmed that this effect is essential for bearing performance. Detailed thermohydrodynamic (THD) analysis has also been conducted to confirm the important role of inertia [22] and superlaminar flow regimes [23] in flow simulation. Considering the surface roughness effect, the 
Reynolds equation has been modified for an accurate THD analysis of the flow [24].

Taniguchi et al. [25] established an experimental apparatus for a tilting-pad journal bearing to obtain the bearing surface pressure and temperature data under variable bearing loads and rotating speeds. In this study, the simulation results of a THD model with the modified Reynolds equations can catch the general characteristics of experimental data, but not accurately. The accurate predictions of superlaminar bearings need precise models for turbulence effects in oil flows. Meanwhile, compared with the Reynolds model, the computational fluid dynamic techniques have enough available turbulence models for oil flow simulations. Therefore, CFD is a feasible method for simulations of superlaminar bearing oil flows.

CFD techniques have been utilized to simulate superlaminar oil flows in bearings. Maneshian and Gandjalikhan Nassab [26] reported the CFD results of a journal bearing with the AKN low-Re $k-\varepsilon$ turbulence model. On the basis of this work, Solghar and Gandjalikhan Nassab [27] numerically analyzed plain full journal bearings with the same turbulence model.

The turbulence models play an important role in CFD simulations and influence the simulation results significantly. While Bouard et al. have compared three turbulence models using Reynolds equation in tilting-pad journal bearings [28], the turbulence models in CFD techniques are different from Reynolds equation and current CFD studies of superlaminar bearings have not provided a detailed comparison of different turbulence models. Thereby, this paper compares temperature and pressure simulation results of fourteen turbulence models with experimental data from Taniguchi et al. [25] to determine the most suitable turbulence model for superlaminar simulation and explain the underlying mechanisms and what is superlaminar flow.

The remaining parts of this paper are organized as follows. Section 2 presents the geometry and meshing of the test superlaminar journal bearing of Taniguchi et al. [25]. Section 3 describes fourteen turbulence models to simulate superlaminar flow. Section 4 discusses the temperature, pressure, and turbulent viscosity ratio and demonstrates the most suitable turbulence model for the THD simulation of superlaminar oil flow. Section 5 compares the simulated velocity distributions and theoretical results based on law of wall. Section 5 further illustrates the mechanism of superlaminar flows and factors contributing to the different performance levels of the turbulence models. Section 6 shows the conclusions on the selections of the turbulence models for superlaminar flows and reveals the physical meaning of superlaminar flows.

\section{Geometry and Meshing}

The simulation object is the test tilting-pad journal bearing of Taniguchi et al. [25]. Figure 1 depicts the cross section of the entire experimental apparatus. Table 1 lists the specifications of this journal bearing.

The simulated working condition for this tilting-pad journal bearing is selected as the standard working condition at $3000 \mathrm{r} / \mathrm{min}$ and under $180 \mathrm{kN}$ loading. Given the
TABLE 1: Specifications of tested bearing [25].

\begin{tabular}{lc}
\hline Bearing type & Tilting pad \\
\hline Bearing diameter $(\mathrm{mm})$ & 479 \\
Bearing length $(\mathrm{mm})$ & 300 \\
Radial clearance $(\mathrm{mm})$ & 0.612 \\
Pad thickness (mm) & 121 \\
Number of pads & 4 \\
Pad arc (dleg) & 80 \\
Pivot offset & $0 \%$ \\
Preload factor & 0 \\
Load angle & Between pads \\
\hline
\end{tabular}

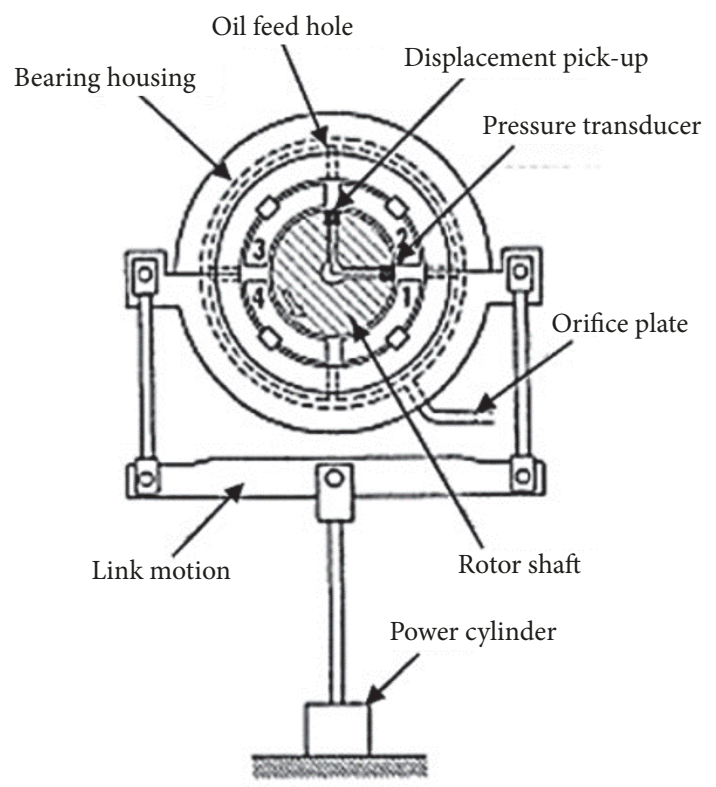

Figure 1: Geometry of the stream surface [25].

experimental film pressure distributions shown in Figure 2, numbers 2 and 3 bearing pads are loaded, whereas numbers 1 and 4 bearing pads are unloaded. The oil flow in number 3 pad has similar characteristics to number 2 pad because of the load angle between the pads. Thus, the entire journal bearing is replaced by one loaded pad in the simulations to simplify the bearing geometry. The main characteristics of the oil flow can also be obtained from the simulation results of the loaded pad.

Figure 3 shows the measured oil film thickness of number $2 \mathrm{pad}$ at $3000 \mathrm{r} / \mathrm{min}$ and under $180 \mathrm{kN}$ loading [25]. The three-dimensional geometry of the structured mesh for oil is fixed based on the measured film thickness and the mesh independence of the structured mesh has been validated [29]. The structured mesh is shown in Figure 4. Along the lubricant flowing direction, the radial thickness is set as the measured film thickness. In the axial direction, the radial thickness is constant and the axial length equals to the bearing length, $300 \mathrm{~mm}$. The side faces are perpendicular to the length direction ( $z$-axis). The rotor-side and pad-side walls are normal to the radial direction. 


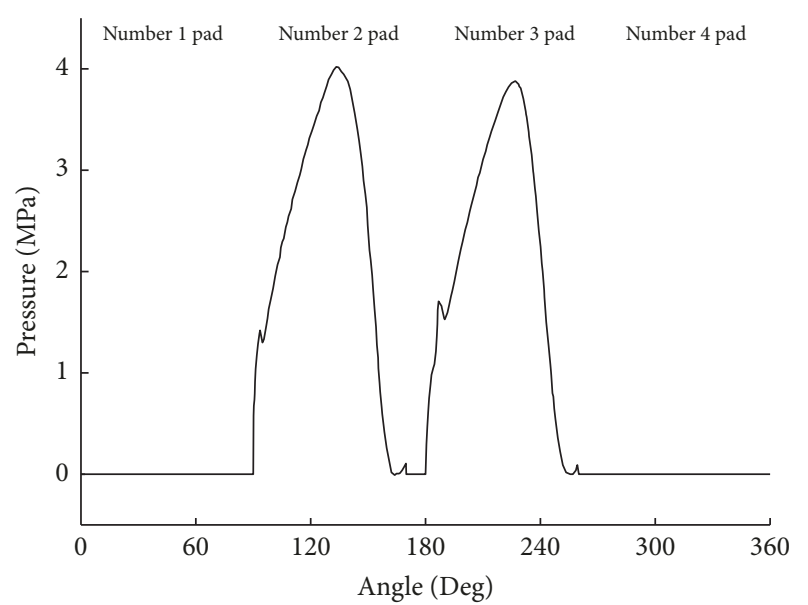

FIGURE 2: Experimental film pressure at $3000 \mathrm{r} / \mathrm{min}$ and under $180 \mathrm{kN}$ loading [25].

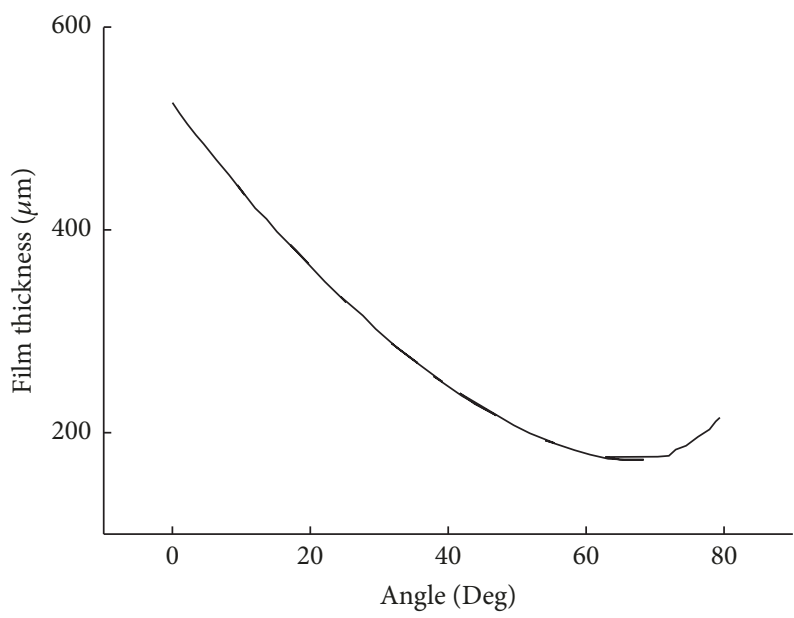

FIGURE 3: Measured film thickness at $3000 \mathrm{r} / \mathrm{min}$ and under $180 \mathrm{kN}$ loading [25].

The minimum orthogonal quality of number 2 pad mesh is equal to 0.757 , where the values near 1 correspond to high quality. The maximum ortho skew of number 2 pad mesh is equal to 0.243 , where the values near 0 correspond to high quality. Therefore, this structured mesh has high quality.

The lubricant for this journal bearing is ISO VG32. Considering the thermal effect on material viscosity, the piecewise linear interpolation method is employed to determine the relationship between the temperature and viscosity with the data listed in Table 2. The supply oil pressure and temperature in the oil feed hole are $0.1 \mathrm{MPa}$ and $315.15 \mathrm{~K}$, respectively, based on the data of the experimental apparatus.

Table 3 lists the boundaries in detail with the reference pressure set as 1 bar. Considering the outlet of number 2 pad is at the position of another oil inlet hole and the measured outlet pressure is $0.1 \mathrm{MPa}$, the relative pressures of the outlet of number 2 pad is set at $0.1 \mathrm{MPa}$. The inlet mixture temperature is assumed to be uniformly distributed, and its value is set at the measured temperature of $315.35 \mathrm{~K}$

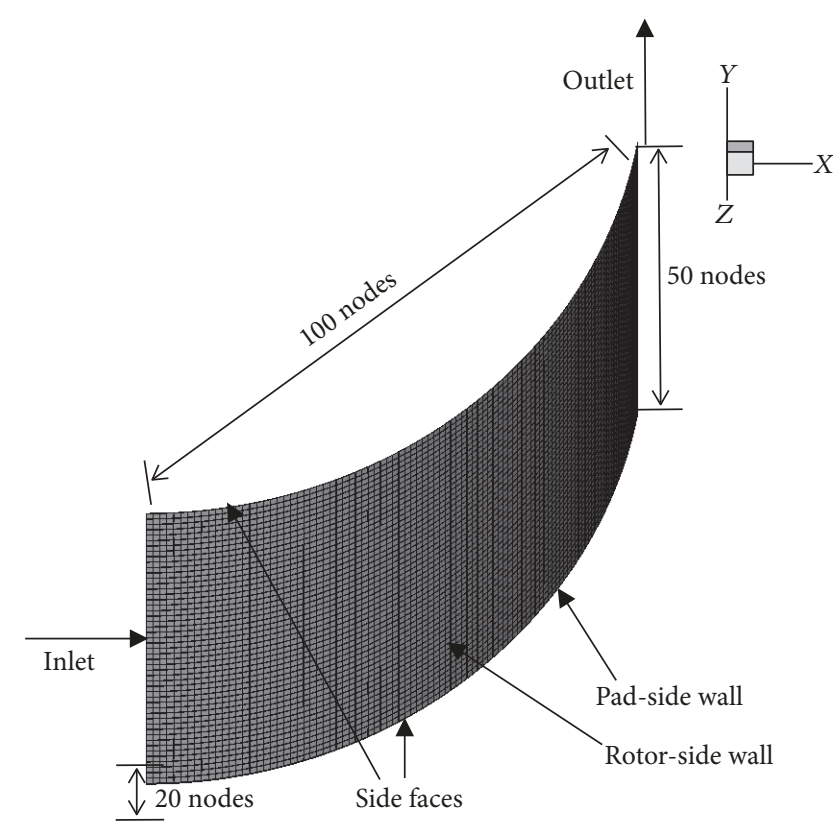

Figure 4: Mesh [29].

TABLE 2: Viscosity properties of ISO VG32.

\begin{tabular}{lc}
\hline Temperature $(\mathrm{K})$ & Viscosity $(\mathrm{kg} /(\mathrm{m} \cdot \mathrm{s}))$ \\
\hline 313.15 & 0.0275 \\
318.15 & 0.0224 \\
323.15 & 0.0185 \\
328.15 & 0.0155 \\
333.15 & 0.0131 \\
338.15 & 0.0112 \\
343.15 & 0.0096 \\
348.15 & 0.0084 \\
353.15 & 0.0073 \\
373.15 & 0.0046 \\
\hline
\end{tabular}

TABLE 3: Boundaries.

\begin{tabular}{lcc}
\hline Boundary & Boundary type & Boundary details \\
\hline Inlet & Pressure inlet & $0.1 \mathrm{MPa} 315.15 \mathrm{~K}$ \\
Outlet & Pressure outlet & $0.1 \mathrm{MPa}$ \\
Side faces & Pressure outlet & $0 \mathrm{MPa}$ \\
Pad-side wall & No slip & $115 \mathrm{~W} /\left(\mathrm{m}^{2} \cdot \mathrm{K}\right)$ \\
Rotor-side wall & No slip & $115 \mathrm{~W} /\left(\mathrm{m}^{2} \cdot \mathrm{K}\right)$ \\
& & $314.159 \mathrm{rad} / \mathrm{s}$ \\
\hline
\end{tabular}

in the inlet of number 2 pad. Furthermore, the heat transfer coefficient for the pad-side and rotor-side walls is set at $115 \mathrm{~W} /\left(\mathrm{m}^{2} \cdot \mathrm{K}\right)$ with the free stream temperature equal to the inlet temperature based on the work of Taniguchi et al. [25]. The lubricant velocity on the rotor-side wall is set as the rotational speed of the rotor, $314.159 \mathrm{rad} / \mathrm{s}$. The lubricant velocity on the pad-side wall is set as zero. 


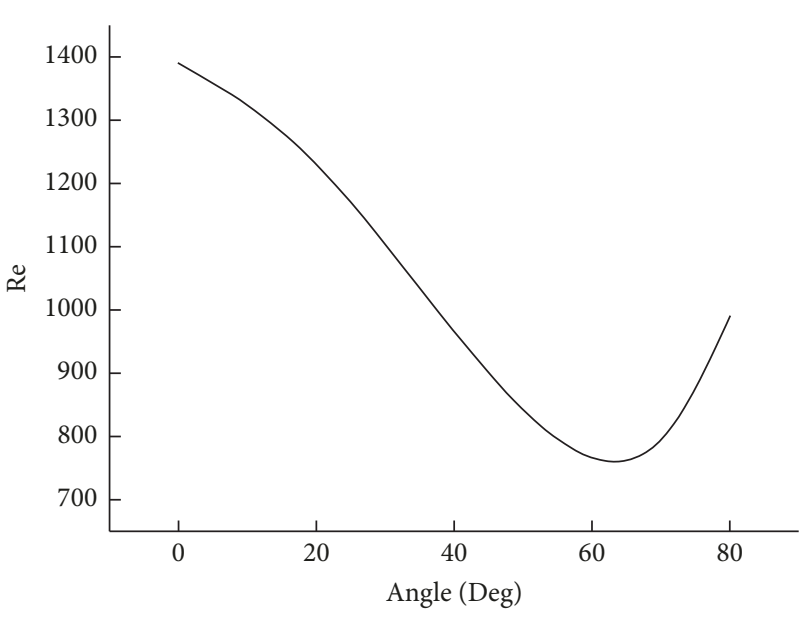

(a)

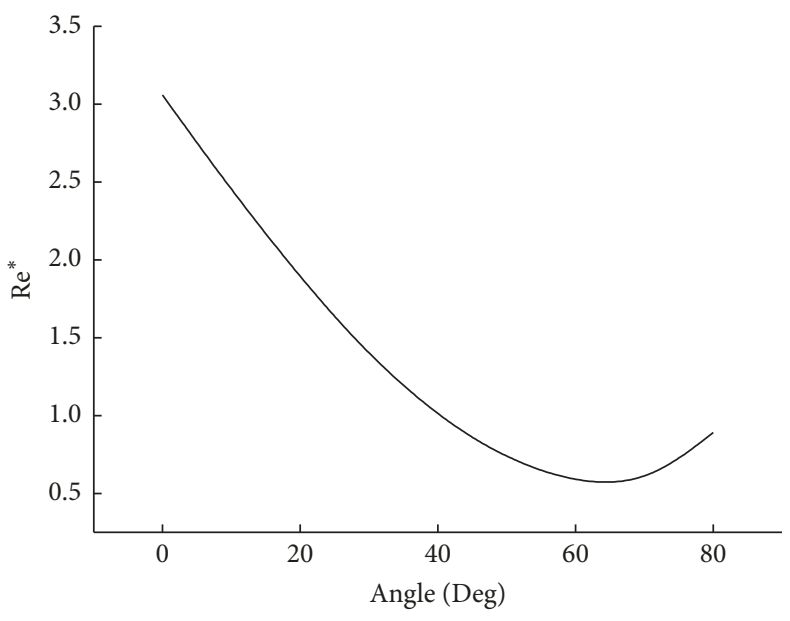

(b)

FIGURE 5: Reynolds number of oil film: (a) Re; (b) Re* .

\section{Turbulence Models}

The oil flow regime in the journal bearing should be discussed clearly before suitable models are selected to facilitate the CFD simulations. This regime can be characterized by the Reynolds number, which can be defined as follows [30]:

$$
\operatorname{Re}=\frac{\rho u d}{\mu}
$$

where $d$ is set as the measured oil film thickness, $u$ is set as the rotational speed of the rotor-side wall, and the value of $\mu$ is decided by the rotor-side temperature to calculate the Reynolds number of the oil film.

Apart from the noted definition for the Reynolds number, an alternative reduced Reynolds number is also typically defined for considering the effect of the shaft diameter properly in bearing analyses [30]. Its definition is

$$
\operatorname{Re}^{*}=\frac{\rho u R}{\mu}\left(\frac{d}{R}\right)^{2}
$$

where $R$ is set as the radius of the rotor.

Both the definitions of the Reynolds number are the accepted methods of nondimensionalizing the flow turbulence characteristics in modern bearing treatments [30]. The calculated Reynolds numbers initially decrease and then increase in outlet (Figure 5). The Reynolds numbers change indicates that the flow regime changes from superlaminar to laminar and turbulence effect on the oil film changes with the Reynolds numbers. Thus, the turbulence effect should be considered in the simulations.

This study uses ANSYS Fluent with finite volumes for the bearing simulations. In CFD, the basis of the incompressible turbulence and laminar models is the Navier-Stokes equation and continuous equation. Navier-Stokes equation is expressed as follows:

$$
\begin{aligned}
\rho\left(\frac{\partial u}{\partial t}+u \cdot \nabla u\right)= & -\nabla p+\rho f+\nabla(\lambda \nabla \cdot u)+\nabla \\
& \cdot\left(2\left(\mu+\mu_{t}\right) E\right) .
\end{aligned}
$$

The continuous equation is

$$
\nabla \cdot u=0
$$

Considering the thermal effect, the energy equation is included:

$$
\rho \frac{D h}{D t}=\frac{D p}{D t}+\nabla \cdot(k \nabla T)+\phi
$$

where the viscous dissipation function is given by the following:

$$
\phi=2\left(\mu+\mu_{t}\right)\left[\left(\frac{\partial u_{i}}{\partial x_{i}}\right)\left(\frac{\partial u_{i}}{\partial x_{i}}\right)+\frac{1}{2}\left(\frac{\partial u_{j}}{\partial x_{l}}\right)\left(\frac{\partial u_{l}}{\partial u_{j}}\right)\right] .
$$

The values of turbulent viscosity $\mu_{t}$ and kinetic viscosity $\mu$ are required to solve the aforementioned equations. While the laminar model considers turbulent viscosity as zero, the turbulence models utilize variable methods to obtain the value of turbulent viscosity.

A total of fourteen turbulence models are employed to simulate the oil flow. Table 4 shows that these models are classified into three groups: the classical fully developed turbulence models, transition turbulence models, and turbulence models with low-Re correction. Each group includes different corrections for the standard turbulence models, which forms the new modified turbulence models.

Different corrections are added to these turbulence models.

The equations of the low-Re corrections for different turbulence models are variable. For example, the purpose 
TABLE 4: Turbulence models.

\begin{tabular}{|c|c|c|}
\hline Classical full-developed turbulence models & Transition turbulence models & $\begin{array}{l}\text { Turbulence models with low-Re } \\
\text { correction }\end{array}$ \\
\hline $\begin{array}{l}\text { (1) SA model } \\
\text { (2) Standard } k-\varepsilon \text { model with scalable wall treatment } \\
\text { (3) RNG } k-\varepsilon \text { model with scalable wall treatment } \\
\text { (4) Realizable } k-\varepsilon \text { model with scalable wall treatment } \\
\text { (5) Standard } k-\omega \text { model } \\
\text { (6) SST model } \\
\text { (7) Quadratic pressure-strain RSM model }\end{array}$ & $\begin{array}{l}\text { (8) SST model with intermittency transition } \\
\text { (9) Transition SST model (Menter-Langtry } \gamma-\theta \\
\text { Laminar-Turbulent Transition) } \\
\text { (10) Transition } k-k l-\omega \text { model }\end{array}$ & $\begin{array}{l}\text { (11) AKN low-Re } k-\varepsilon \text { model } \\
\text { (12) Abid low-Re } k-\varepsilon \text { model } \\
\text { (13) Lam-Bremhorst low-Re } k-\varepsilon \\
\text { model } \\
\text { (14) SST model with low-Re } \\
\text { correction }\end{array}$ \\
\hline
\end{tabular}

TABLE 5: Parameters of $k-\varepsilon$ models.

\begin{tabular}{|c|c|c|c|c|c|c|}
\hline$k-\varepsilon$ models & $C_{\mu}$ & $C_{1 \varepsilon}$ & $C_{2 \varepsilon}$ & $C_{2}$ & $\sigma_{k}$ & $\sigma_{\varepsilon}$ \\
\hline Standard $k-\varepsilon$ (scalable wall treatment) & \multirow{4}{*}{0.09} & \multirow{4}{*}{1.44} & \multirow{4}{*}{1.92} & & \multirow{4}{*}{1} & \multirow{4}{*}{1.3} \\
\hline AKN $k-\varepsilon$ (low-Re correction) & & & & & & \\
\hline Abid $k-\varepsilon$ (low-Re correction) & & & & & & \\
\hline Yang-Shih $k-\varepsilon$ (low-Re correction) & & & & & & \\
\hline RNG $k-\varepsilon$ (scalable wall treatment) & 0.0845 & 1.42 & 1.68 & & & \\
\hline Realizable $k-\varepsilon$ (scalable wall treatment) & & 1.44 & & 1.9 & 1 & 1.2 \\
\hline
\end{tabular}

of the low-Re correction for the SST model is to dampen the turbulent viscosity with the coefficient $\alpha^{*}$ and modify the transport equations of the turbulent kinetic energy and specific dissipation rate. The $\alpha^{*}$ equation is expressed as follows:

$$
\alpha^{*}=\frac{0.144+\mathrm{Re}_{t}}{6+\mathrm{Re}_{t}}, \quad \mathrm{Re}_{t}=\frac{\rho k}{\mu \omega} .
$$

The intermittency transition correction is based on the Menter-Langtry $\gamma-\theta$ laminar-turbulent transition model, which is also called the transition SST model. The modifications in the production and destruction terms from the turbulent kinetic energy equation are implemented to ensure that the kinetic energy production is sufficiently low at the transition point.

All of the turbulence models in Table 4 have adjustable parameters. These parameters are set to their default values, which are the same as those in the FLUENT software, to set the turbulence models reasonably. For example, the parameters of the $k-\varepsilon$ models are listed in Table 5 .

All turbulence models use the half-Sommerfeld cavitation model.

\section{Simulation Result Comparisons}

4.1. Temperature Distribution Comparisons. An accurate simulation of temperature distributions is necessary to determine the THD characteristics. Therefore, the simulated temperature distributions in the pad-side center line are selected for comparison with the experimental data of number 3 pad from the results of Taniguchi et al. [25] to determine the most suitable turbulence model for superlaminar journal bearing simulation.

Compared with the experimental data, Figure 6 shows the temperature simulation results in the pad-side center line.
Figure 6 shows that the simulated pad-side temperature of the classical fully developed turbulence models is much lower than the experimental data. The temperature difference around the outlet is more than $5 \mathrm{~K}$. By contrast, the laminar pad-side temperature is $5 \mathrm{~K}$ higher than the experimental temperature data around the outlet. Regardless of the high or low temperature simulation results, remarkable differences from the experimental data indicate that the classical fully developed turbulence models and laminar model cannot accurately determine the thermodynamic characteristics of the superlaminar oil flow.

Apart from the classical fully developed turbulence models, the performance of transition turbulence models is poor. Among them, the transition $k-k l-\omega$ model and SST model with intermittency transition model have almost the same temperature simulation results as the laminar model. The least error of the transition turbulence models is still more than $5 \mathrm{~K}$ around the outlet. Therefore, the transition turbulence models are unsuitable for the THD simulation of the superlaminar bearing.

Although the temperature distributions of the $k-\varepsilon$ models with low-Re correction are approximately $5 \mathrm{~K}$ higher than the experimental data, the SST model with low-Re correction has almost the same temperature simulation results as the experimental data. Its largest error is approximately $2 \mathrm{~K}$, which is lower than the errors of the other thirteen turbulence models. Thus, considering that temperature distribution is a vital thermal characteristic, the SST model with low-Re correction is the best turbulence model for the superlaminar oil flow simulation.

4.2. Load Comparisons. In the numerical simulations, the film geometry of this journal bearing is given by the experimental film thickness distribution under $180 \mathrm{kN}$ and at $3000 \mathrm{r} / \mathrm{min}$. Considering the bearing load is a vital characteristic, the simulated bearing loads are compared with the experimental load. 


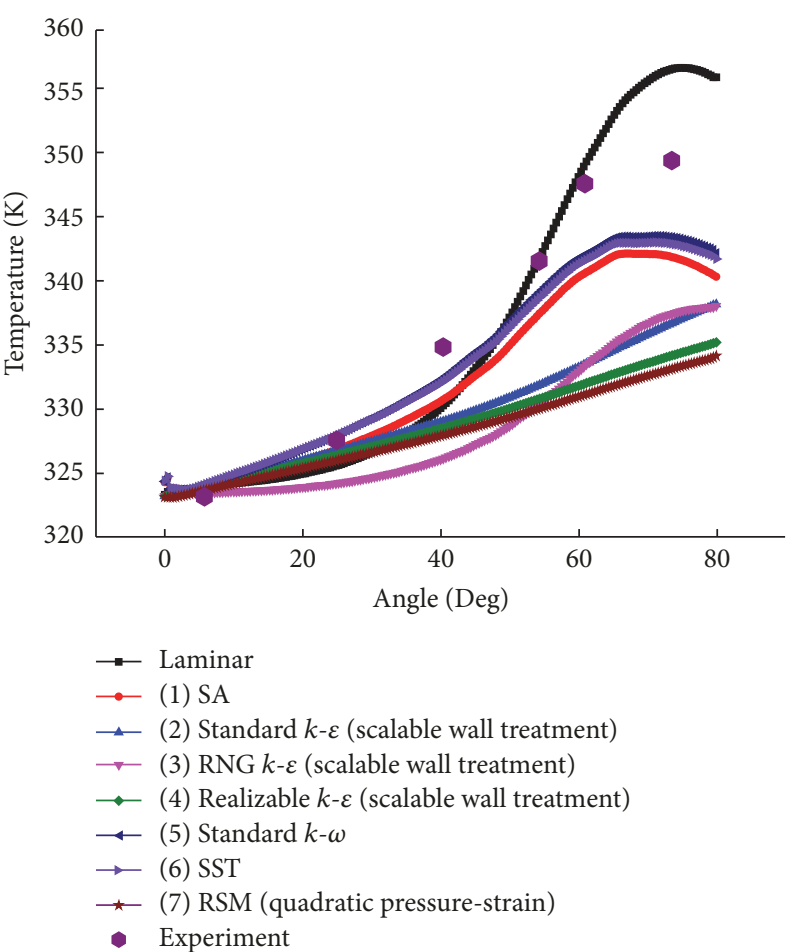

(a)

- Experiment

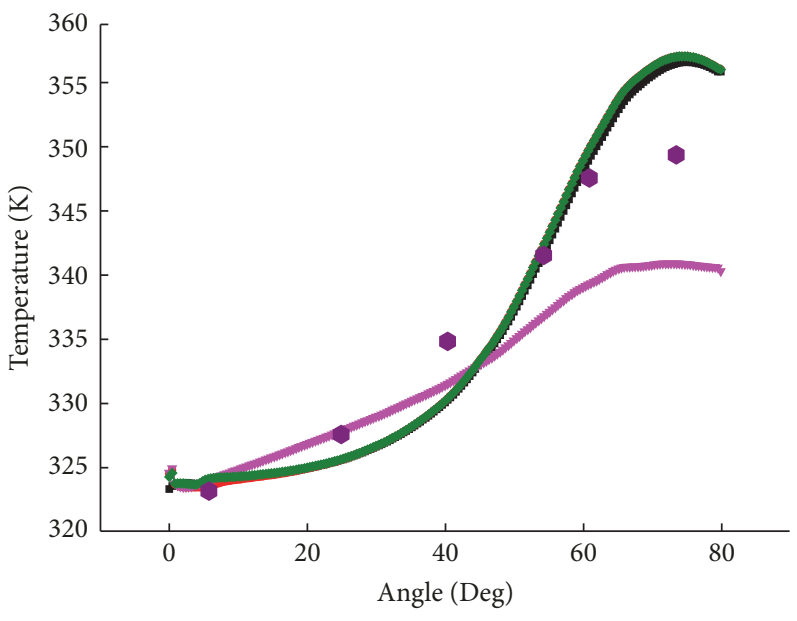

- Laminar

- (8) SST (intermittency transition model)

$\longrightarrow$ (9) Transition SST (Menter-Langtry $\gamma-\theta$ laminar-turbulent transition)

$\rightarrow$ (10) Transition $k-k l-\omega$

- Experiment

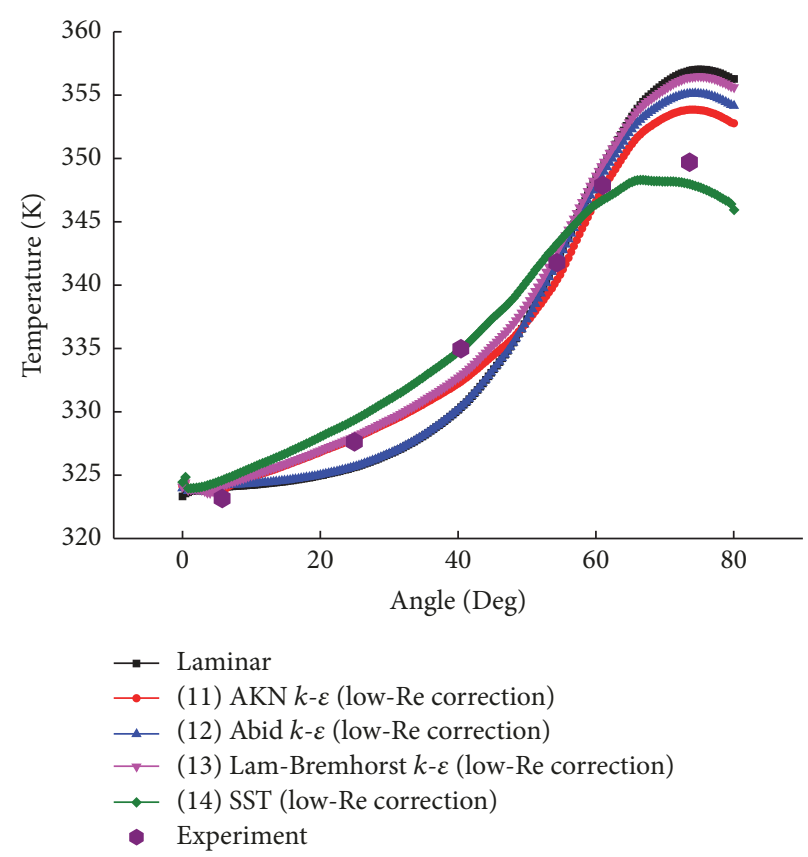

(b)

(c)

FIGURE 6: Comparison between the experimental temperature profile at $3000 \mathrm{rpm}$ and under $180 \mathrm{kN}$ and simulated temperature profile with the fixed film geometry in pad-side center line for (a) the group of the classical turbulence models; (b) the group of the transition turbulence models; and (c) the group of the turbulence models with low-Re correction.

As noted in Section 2 and Figure 2, the loaded pads provide load-bearing and the loaded pads, numbers 2 and 3 pads, have almost the same pressure distribution. Thus, one loaded pad provides the half bearing load, equaling to
$90 \mathrm{kN}$. The pad loads simulated by different models are listed in Table 6.

As shown in Table 6, the pad load simulated by the SST model with low-Re correction is closest to the experimental 
TABLE 6: Pad loads.

\begin{tabular}{|c|c|c|c|}
\hline Group & Model & Corrections & Pad loads $(\mathrm{kN})$ \\
\hline Classical turbulence models & laminar & & 54.2 \\
\hline \multirow{3}{*}{ Classical turbulence models } & SA & & 79.8 \\
\hline & Standard $k-\omega$ & - & 48.2 \\
\hline & SST & & 58.5 \\
\hline \multirow{3}{*}{ Classical turbulence models } & Standard $k-\varepsilon$ & & 34.2 \\
\hline & RNG $k-\varepsilon$ & Scalable wall treatment & 22.3 \\
\hline & Realizable $k-\varepsilon$ & & 37.6 \\
\hline Classical turbulence models & Quadratic pressure-strain RSM & - & 35.2 \\
\hline \multirow{2}{*}{ Transition turbulence models } & Transition SST & \multirow{2}{*}{-} & 58.8 \\
\hline & Transition $k-k l-\omega$ & & 54.2 \\
\hline Transition turbulence models & SST & Intermittency transition & 54.5 \\
\hline \multirow{3}{*}{ Turbulence models with low-Re correction } & AKN $k-\varepsilon$ & \multirow{3}{*}{ Low-Re correction } & 58.4 \\
\hline & Abid $k-\varepsilon$ & & 56.6 \\
\hline & Lam-Bremhorst $k-\varepsilon$ & & 57.5 \\
\hline Turbulence models with low-Re correction & SST & Low-Re correction & 88.9 \\
\hline Experiment & - & - & 90.0 \\
\hline
\end{tabular}

data and its relative error is $1.27 \%$, accurate enough for the bearing load predictions.

Among the classical turbulence models, the SA model has the best pad load and its error is still larger than 10\%, unacceptable for the load predictions. As for the transition turbulence models and other turbulence models with lowRe correction, their errors are all larger than 33\% and cannot predict the load precisely. Thus, among all these models, the SST model with low-Re correction is the only suitable one for the bearing load simulations.

4.3. Pressure Distribution Comparisons. In addition to temperature distributions, the simulation of the pressure distributions should be corrected to obtain accurate THD characteristics. The simulated pad-side pressure distributions in the pad-side center line are compared with the experimental data from Taniguchi et al. [25] to determine the most suitable turbulence model for the superlaminar journal bearing simulation.

4.3.1. Classical Turbulence Models. Compared with the experimental data, the pressure simulation results of the classical turbulence models in the center line of the pad-side wall are shown in Figure 7.

The highest simulated pad-side pressure of laminar is $2.43 \mathrm{MPa}$ at $40.0^{\circ}$ from the inlet, which is $39.6 \%$ lower than the highest measured pad-side pressure of $4.02 \mathrm{MPa}$ at $43.3^{\circ}$. Although the simulation error for the angle of the highest pad-side pressure is acceptable, the error for the highest pressure value cannot be neglected. Thus the turbulence effect on the hydrodynamic characteristics is not negligible.

Table 7 shows that the errors in the highest pressure values for the classical fully developed turbulence models are more than $20 \%$. The differences with experimental data are unacceptable. Thus, the turbulent effects in the superlaminar oil flow should be considered different from the fully developed turbulent flow.

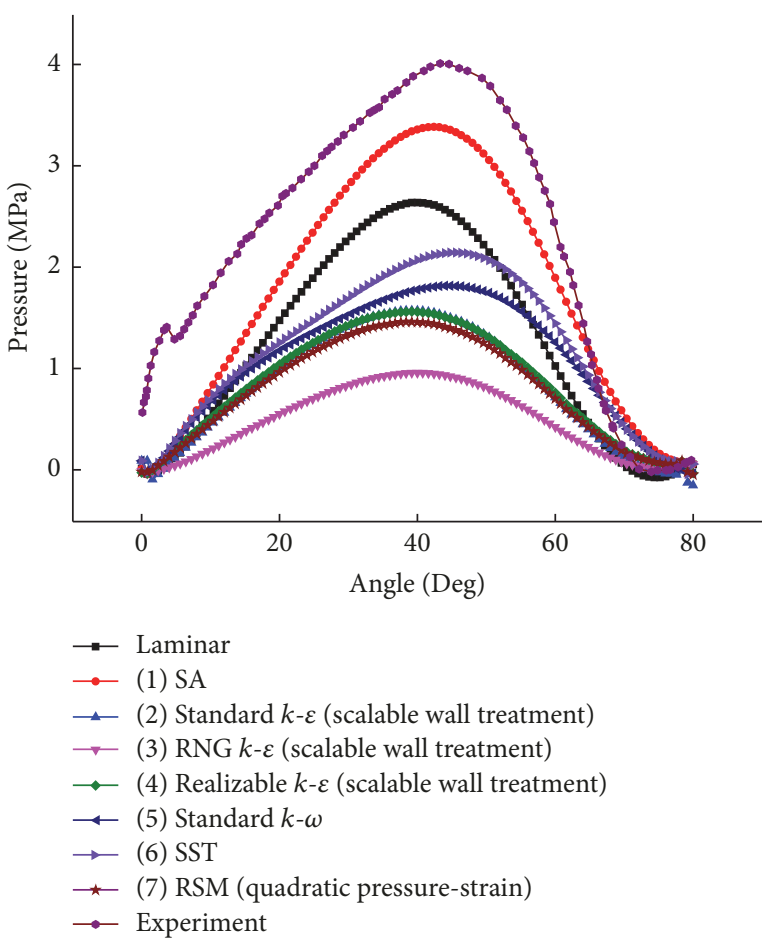

FIGURE 7: Comparison between the experimental pressure profile at $3000 \mathrm{rpm}$ and under $180 \mathrm{kN}$ and the simulated pressure profiles with the fixed film geometry in pad-side center line for the group of the classical turbulence models.

A small difference is observed among the simulation results of the different classical $k-\varepsilon$ models. Compared with the RNG $k-\varepsilon$ model with scalable wall treatment, the simulation results of the realizable $k-\varepsilon$ and standard $k-\varepsilon$ models with scalable wall treatment are more consistent with the experimental pressure data, which are still much worse than the laminar results. 
TABLE 7: Highest pressure of the classical full-developed turbulence models group.

\begin{tabular}{lccc}
\hline & Highest pressure & Value & $\begin{array}{c}\text { Position } \\
(\text { Degree })\end{array}$ \\
Model & Corrections & (MPa) & 41.6 \\
\hline SA & & 3.09 & 4.83 \\
Standard $k-\omega$ & - & 2.15 & 45.6 \\
SST & Scalable wall treatment & 1.58 & 39.2 \\
\hline Standard $k-\varepsilon$ & & 0.97 & 40.0 \\
RNG $k-\varepsilon$ & & 1.57 & 39.2 \\
Realizable $k-\varepsilon$ & & 1.47 \\
\hline Quadratic pressure-strain RSM & & 39.2 \\
\hline
\end{tabular}

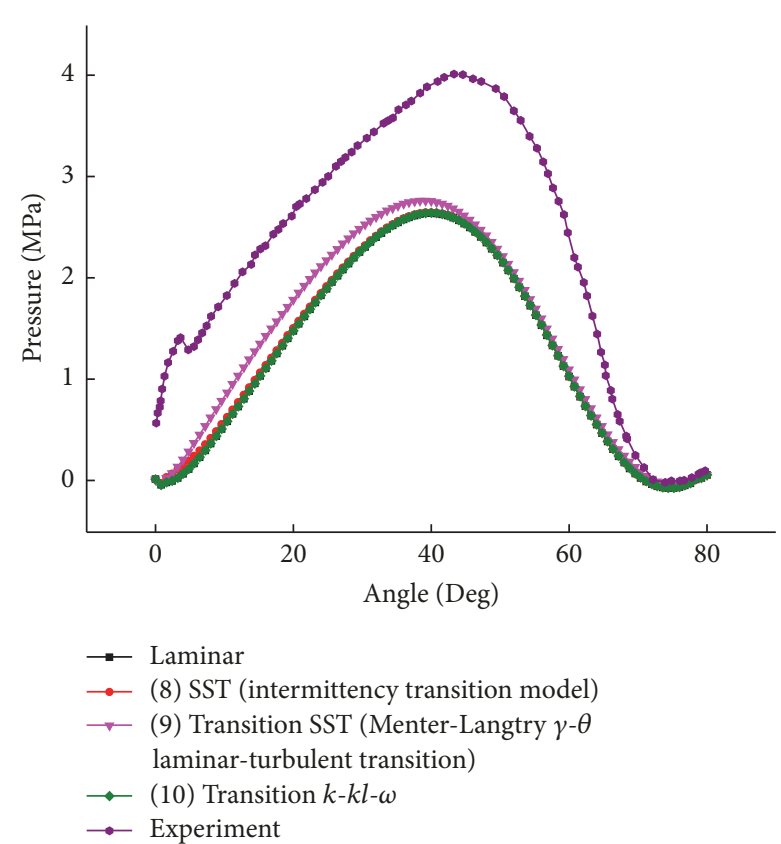

FIGURE 8: Comparison between the experimental pressure profile at $3000 \mathrm{rpm}$ and under $180 \mathrm{kN}$ and the simulated pressure profiles with the fixed film geometry in pad-side center line for the group of the transition turbulence models.

4.3.2. Transition Turbulence Models. Compared with the experimental data, the pressure simulation results of the transitional turbulence models in the center line of the pad-side wall are shown in Figure 8.

Table 8 shows that the highest pressure value and angle of the transition SST model are 31.09\% lower and 9.47\% earlier than the experimental data, which are the best results in the transition turbulence models. However, these values are still unacceptable for the precious bearing simulation.

The transition $k-k l-\omega$ model and the SST model with intermittency transition model perform worse than several classical fully developed turbulence models, such as SA model. Thus, the transition model is unsuitable for the simulations of superlaminar oil flow and other corrections should be added to the turbulence models for this simulation.

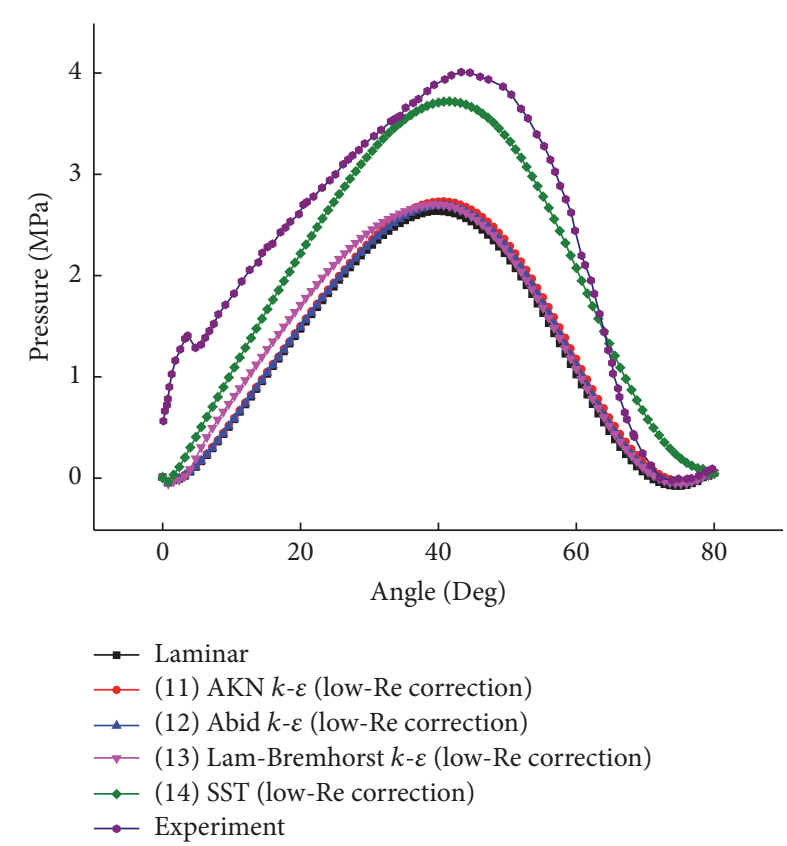

Figure 9: Comparison between the experimental pressure profile at $3000 \mathrm{rpm}$ and under $180 \mathrm{kN}$ and the simulated pressure profiles with the fixed film geometry in pad-side center line for the group of turbulence models with low-Re correction.

4.3.3. Turbulence Models with Low-Re Correction. Compared with the experimental data, the pressure simulation results of the turbulence models with low-Re correction in the center line of the pad-side wall are shown in Figure 9.

Table 9 shows that the simulation results vary for the different turbulence models, and the best model in this group is the SST model with low-Re correction. The error of the highest pad-side pressure value for this model is $7.21 \%$, which is also the best among the fourteen turbulence models.

Compared with the theoretical data [25], the highest pressure value of the SST model with low-Re correction is higher and has a smaller difference with the experimental data. The pressure simulated by SST model with low-Re correction around the outlet decreases faster and is nearer to the change tendency of the experimental data than the theoretical data. Furthermore, the theoretical data is obtained by solving the modified Reynolds equations, which is different from the 
TABLE 8: Highest pressure of the transition turbulence models group.

\begin{tabular}{lccc}
\hline & \multicolumn{2}{c}{ Highest pressure } & Palue \\
Model & Corrections & (MPa) & $\begin{array}{c}\text { Position } \\
\text { (Degree) }\end{array}$ \\
\hline Transition SST & - & 2.77 & 39.2 \\
Transition $k-k l-\omega$ & - & 2.65 & 40.0 \\
\hline SST & Intermittency transition & 2.65 & 40.0 \\
\hline
\end{tabular}

TABLE 9: Highest pressure of the turbulence models with low-Re correction group.

\begin{tabular}{lccc}
\hline Model & \multicolumn{1}{c}{$\begin{array}{c}\text { Highest pressure } \\
\text { Corrections }\end{array}$} & $\begin{array}{c}\text { Value } \\
(\mathrm{MPa})\end{array}$ & $\begin{array}{c}\text { Position } \\
\text { (Degree) }\end{array}$ \\
\hline AKN $k-\varepsilon$ & & 2.74 & 40.8 \\
Abid $k-\varepsilon$ & Low-Re correction & 2.71 & 40.0 \\
Lam-Bremhorst $k-\varepsilon$ & & 2.71 & 19.2 \\
\hline SST & Low-Re correction & 3.73 & 41.6 \\
\hline
\end{tabular}

CFD methods. Hence, the CFD simulation with the SST model with low-Re correction provides a relevant method to simulate the characteristics of the bearing oil flow.

Meanwhile, the low-Re AKN $k-\varepsilon$ model, utilized by Solghar and Gandjalikhan Nassab [27] and Maneshian and Gandjalikhan Nassab [26], simulates the pressure distribution similar to laminar in this case. This scenario implies that the turbulence effect is not considered suitably by the low-Re AKN $k$ - $\varepsilon$ effect, and the corrections for the transport equations and turbulence viscosity should be modified for the superlaminar flow. Compared with the SST model with low-Re correction, the low-Re $k-\varepsilon$ model generally performs even worse than the simulation results of the standard $k-\varepsilon$ model with scalable wall treatment. This condition indicates that the low-Re correction for $k-\varepsilon$ turbulence models may be unsuitable for the bearing oil flow simulation.

The pad-side pressure contours of the laminar and typical turbulence models in each group are shown in Figure 10. The left border is the oil inlet, whereas the right border is the oil outlet.

The aforementioned contours show that the highest pressure positions are almost the same, which are consistent with the pad-side pressure results in center line and reflects the necessity to analyze the simulated center line pressure.

Therefore, the aforementioned pressure analysis reveals that, among these fourteen turbulence models and laminar model, the SST model with low-Re correction performs the best pressure simulation.

The SST model with low-Re correction can simulate the THD characteristics of superlaminar oil flow accurately based on the pressure and temperature analysis.

4.4. Turbulent Viscosity Ratio Distribution Comparison. The turbulence strength should also be discussed for a detailed comparison. Figure 11 shows the turbulent viscosity ratio profile in center line between the pad-side wall and rotor-side wall. The definition of the turbulent viscosity ratio is the ratio of the turbulent viscosity to laminar viscosity $\left(\mu_{t} / \mu\right)$, which is directly proportional to the turbulent Reynolds number and represents the turbulence strength.

Figure 11 shows a significant increase in the turbulent viscosity ratio for almost all turbulence models around the inlet. This scenario is due to the automatic adjustment to the turbulent kinetic energy, turbulent dissipation rate, and specific dissipation rate of the inlet boundary in the turbulence models.

By ignoring the inlet increase, compared with the Reynolds number of oil flow in Figure 5, the turbulent viscosity ratios of the SST model with low-Re correction and SST model show a similar change tendency of decreasing first and then increasing. This condition means that both of them can catch the change tendency of turbulence effect properly.

The low-Re AKN $k-\varepsilon$ model and SST model with intermittency transition simulate the oil flow as pure laminar with the turbulent viscosity ratio equal to zero. The MenterLangtry $\gamma-\theta$ laminar-turbulent transition model also simulates the oil flow as laminar around the outlet, which explains why transition models exhibit similar temperature distributions to the laminar. Therefore, transition models are unsuitable for the superlaminar flow.

Figure 12 depicts the contours of the turbulent viscosity ratio simulated by the SST model with low-Re correction. The upper boundary is the rotor-side, whereas the lower boundary is the pad-side. The angle of number 2 pad is $80^{\circ}$, and the three selected locations are $20^{\circ}, 40^{\circ}$, and $60^{\circ}$ from the inlet.

Figure 12 shows that the turbulent viscosity ratio simulated by the SST model with low-Re correction decreases slowly from $20^{\circ}$ to $60^{\circ}$, which is consistent with the change tendency in the turbulence viscosity ratio in Figure 11. The contours are symmetric with two low turbulent viscosity ratio regions, which reflect the influence of the two wall boundaries.

These results generally infer that the SST model with lowRe correction produces better results for superlaminar flows 

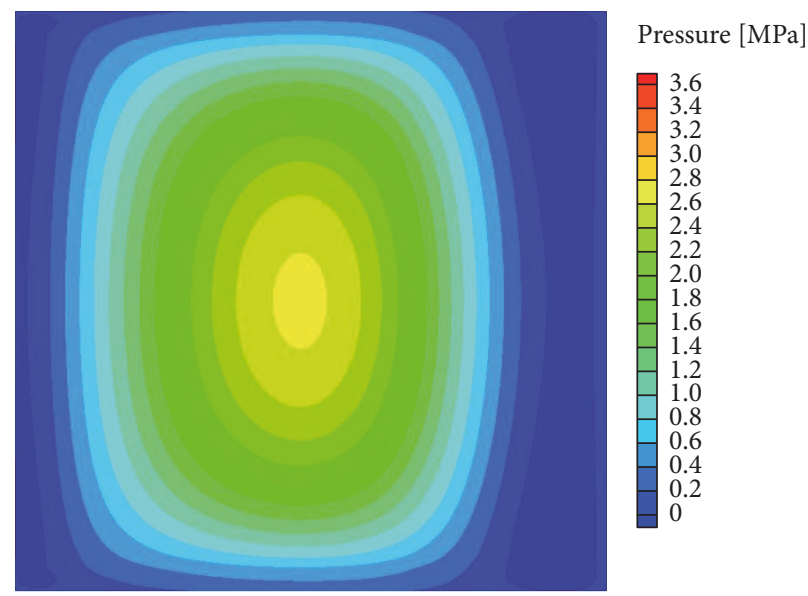

(a)

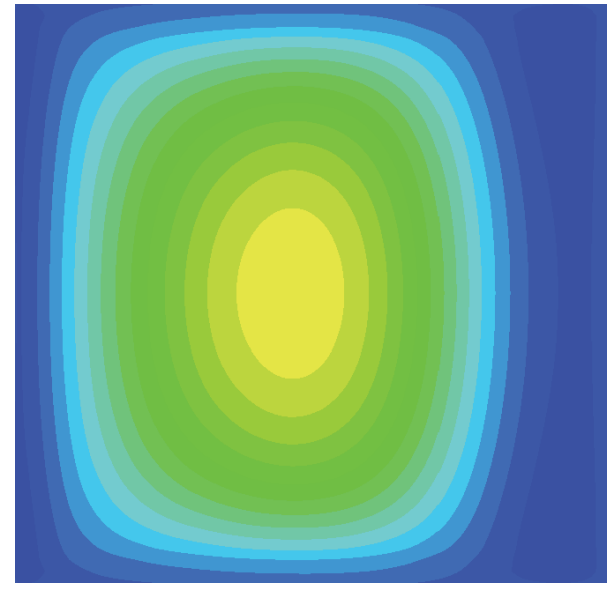

Pressure $[\mathrm{MPa}]$

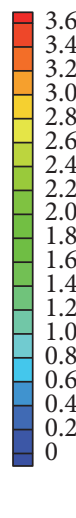

(c)

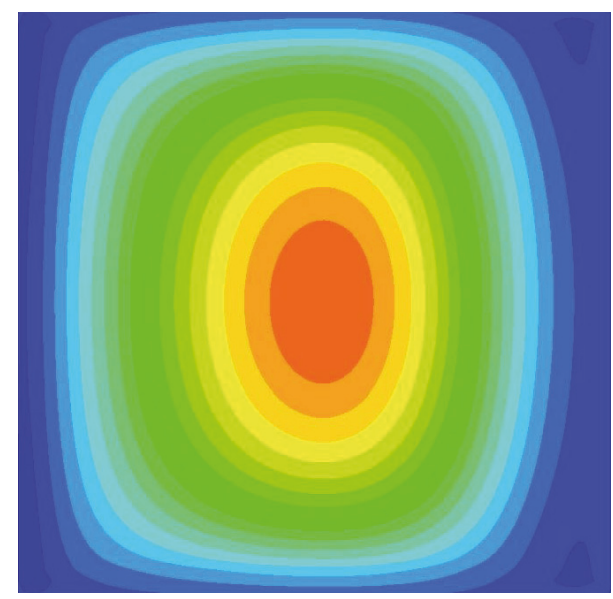

Pressure [MPa]

(b)

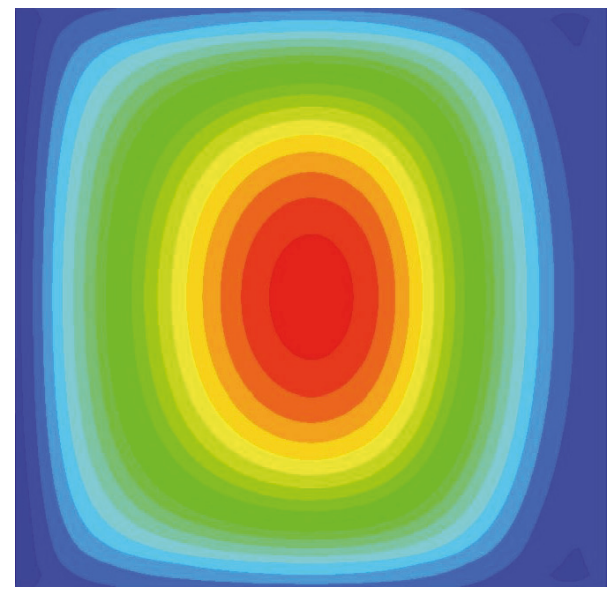

Pressure $[\mathrm{MPa}]$

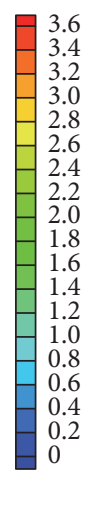

(d)

FIgURE 10: Contours of pad-side pressure. (a) Laminar model; (b) SA model; (c) transition SST model; and (d) SST model with low-Re correction.

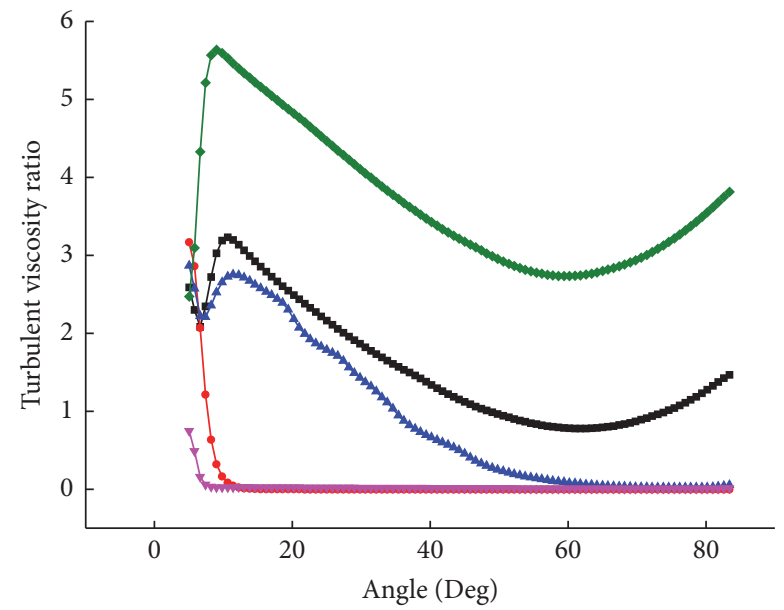

$\rightarrow(6)$ SST

- (8) SST (intermittency transition model)

$\simeq$ (10) Transition SST (Menter-Langtry $\gamma-\theta$ laminar-turbulent transition)

$\rightarrow(12) \mathrm{AKN} k-\varepsilon$ (low-Re correction)

$\rightarrow$ (15) SST (low-Re correction)

FIGURE 11: Turbulent viscosity ratio in center line. in the bearing compared with the other thirteen turbulence models and laminar model.

\section{Velocity Profile Analysis}

The oil flow field should be examined in detail to analyze the SST model with low-Re correction. First, a middle plane, which is perpendicular to the length direction and located in the middle of the pad length, is drawn. Five profiles at different degrees from the inlet are then located in the middle plane. The five velocity profiles are shown in Figure 13.

The difference among the velocity profiles is most evident at $40^{\circ}$ from the inlet, which also shows the influence of the boundary layers. The laminar velocity profile is a straight line from the pad-side wall to the rotor-side wall, whereas the velocity profiles of the SST model and SST model with lowRe correction are S-shaped curves. The S-shaped curves show that the two SST models consider the viscous sublayer and buffer layer. The rotor-side boundary layer also interacts with the pad-side boundary layer in the middle buffer layer region.

The velocity profiles of the three models for $0^{\circ}, 20^{\circ}$, $60^{\circ}$, and $80^{\circ}$ from the inlet do not have such a significant 


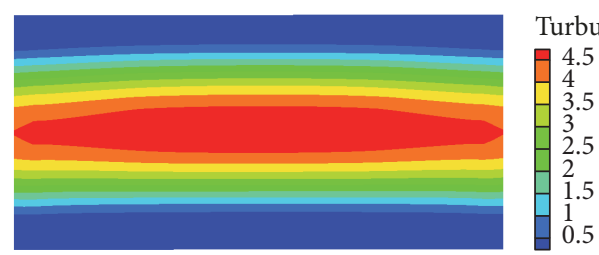

(a)

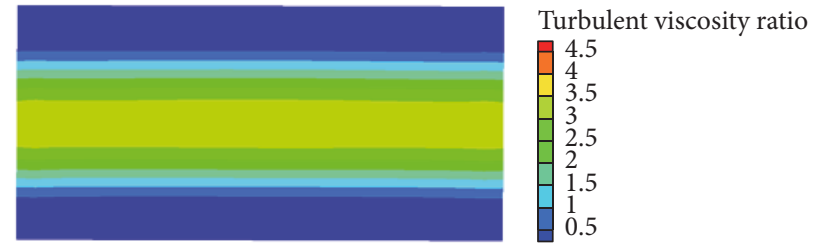

(b)

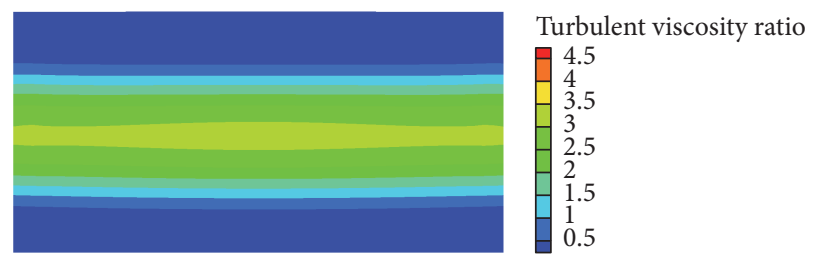

(c)

FIGURE 12: Contours of turbulent viscosity ratio in the sections simulated by SST model with low-Re correction. (a) $20^{\circ}$ from the inlet; (b) $40^{\circ}$ from the inlet; and (c) $60^{\circ}$ from the inlet.

difference from the velocity as the 40 degrees from the inlet, which may be caused by the influence of the inlet and the outlet boundary and cannot show the difference among the velocity profiles of the three models.

However, Figure 13 shows that the velocity profile difference between the SST model with low-Re correction and SST model is not sufficiently clear to analyze the two turbulence models. Thus, the dimensionless parameters $u^{+}$and $y^{+}$are adopted to present the velocity distribution.

Considering that the difference between the velocity profiles is most evident at $40^{\circ}$ from the inlet, then the locations for $u^{+}$and $y^{+}$are set at $40^{\circ}$ from the inlet.

Hence, $y^{+}$can be calculated as follows:

$$
y^{+}=\frac{\rho y u_{\tau}}{\mu}
$$

where $u_{\tau}$ can be calculated as follows:

$$
u_{\tau}=\sqrt{\frac{\tau_{w}}{\rho}}
$$

and $u^{+}$can be calculated as follows:

$$
u^{+}=\frac{u}{u_{\tau}}
$$

Given that $y^{+}$requires the distance value to the nearest wall $(y)$, then the oil flow region is divided into two parts: the pad-side and the rotor-side parts. The dividing line is in the middle of the flow region. The rotor-side and the padside parts utilize the rotor-side and the pad-side wall shear stresses $\left(u_{\tau}\right)$, respectively, to address the two boundary layers individually.

Figure 14 shows that the change tendency of $u^{+}$with $y^{+}$ increasing at $40^{\circ}$ from the inlet. The viscous sublayer and the log-law lines are drawn for comparison with the CFD results based on the $u^{+}$and $y^{+}$functions in the viscous sublayer and the log-law region.
The function of $u^{+}$and $y^{+}$in the viscous sublayer is expressed as follows:

$$
u^{+}=y^{+} \text {. }
$$

The function of $u^{+}$and $y^{+}$in the log-law region for a smooth wall is expressed as follows [31]:

$$
u^{+}=\frac{1}{0.41} \ln y^{+}+5 \text {. }
$$

The pad-side and rotor-side parts of the SST model with low-Re correction obtain similar results. The simulated $u^{+}$ is initially almost the same as the viscous sublayer, which means that this flow region is actually the viscous sublayer. The simulated $u^{+}$is then lower than the viscous sublayer and becomes near the log-law in the end. This scenario means that the boundary layer changes from the viscous sublayer to the buffer layer at the latter part. If sufficient space is available for the boundary layer to be fully developed, then the simulated $u^{+}$can be the same as the log-law at the latter part.

A relatively large difference exists between the pad-side and rotor-side parts for the SST model in terms of value. However, the change tendencies of the simulated $u^{+}$in two parts are almost the same. The simulated $u^{+}$change is initially similar to the viscous sublayer, which means that the viscous sublayer is correctly considered in this turbulence model. However, the simulated $u^{+}$then becomes higher and increases faster than the log-law, which are inconsistent with the law of the wall. This scenario means that the buffer layer is incorrectly considered in the SST model.

Therefore, the buffer layer plays the most important role for the turbulence boundary layer of the superlaminar bearing. This condition indicates that the mechanism of superlaminar flows is near-all turbulence. The classical fully developed turbulence models cannot predict the buffer layer accurately, but an appropriate low-Re correction can address this problem. This scenario explains why the SST model with low-Re correction produces suitable results for superlaminar flows in the bearing. 

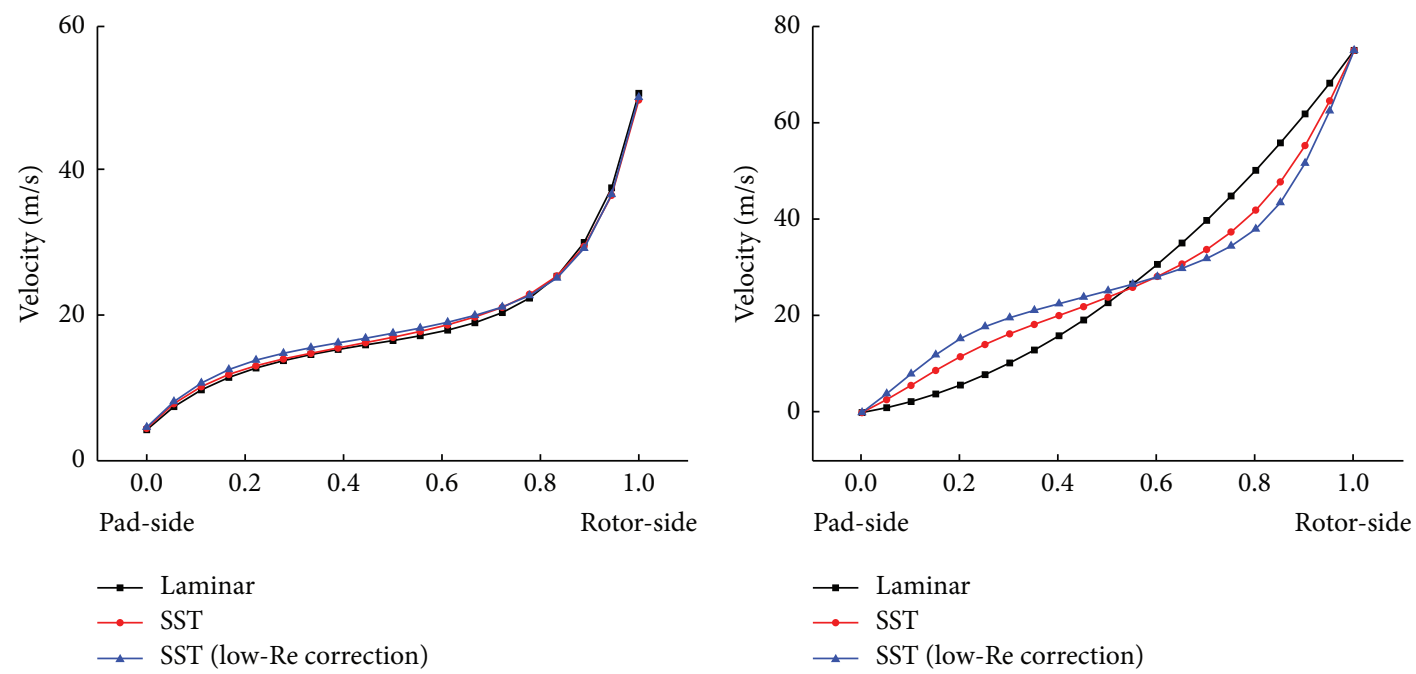

(a)

(b)

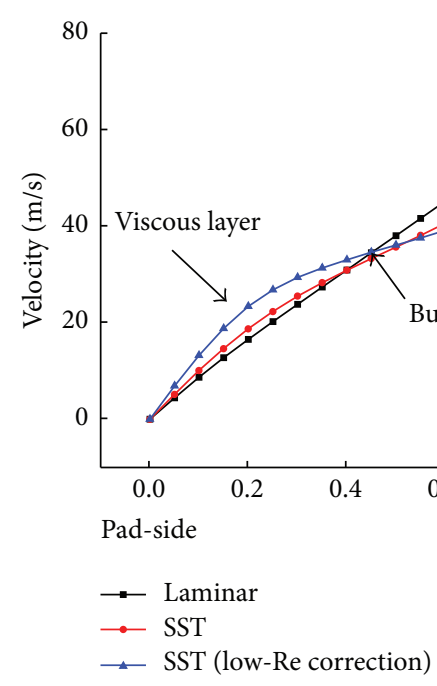

(c)

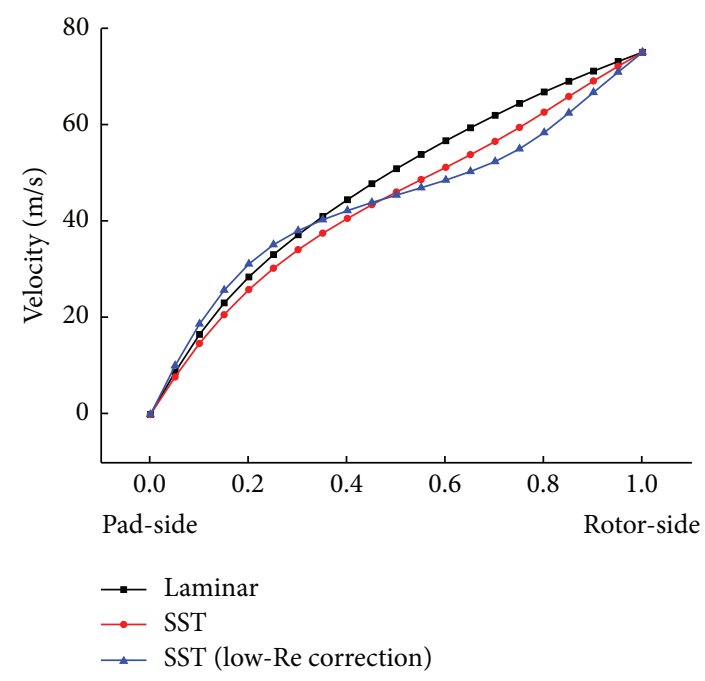

(d)

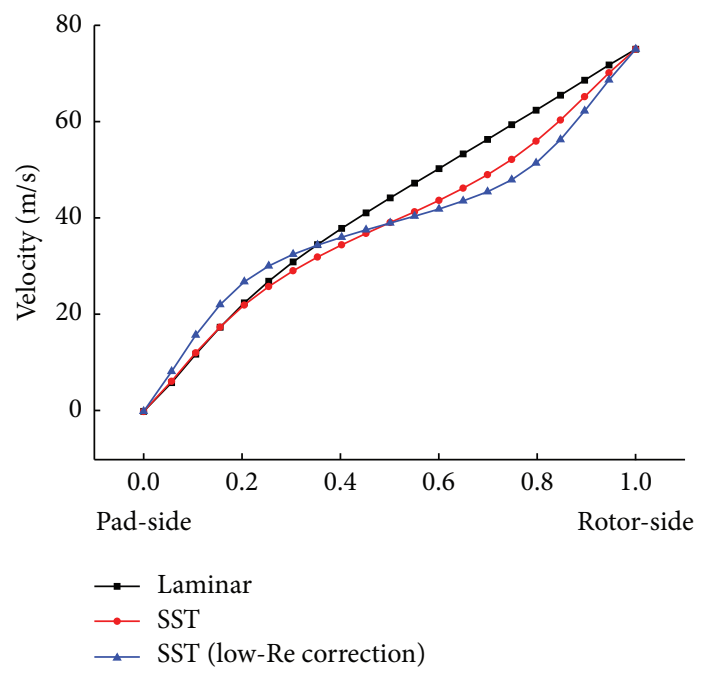

(e)

Figure 13: Velocity profiles in the middle plane. (a) In $0^{\circ}$ from the inlet; (b) in $20^{\circ}$ from the inlet; (c) in $40^{\circ}$ from the inlet; (d) in $60^{\circ}$ from the inlet; and (e) in $80^{\circ}$ from the inlet. 


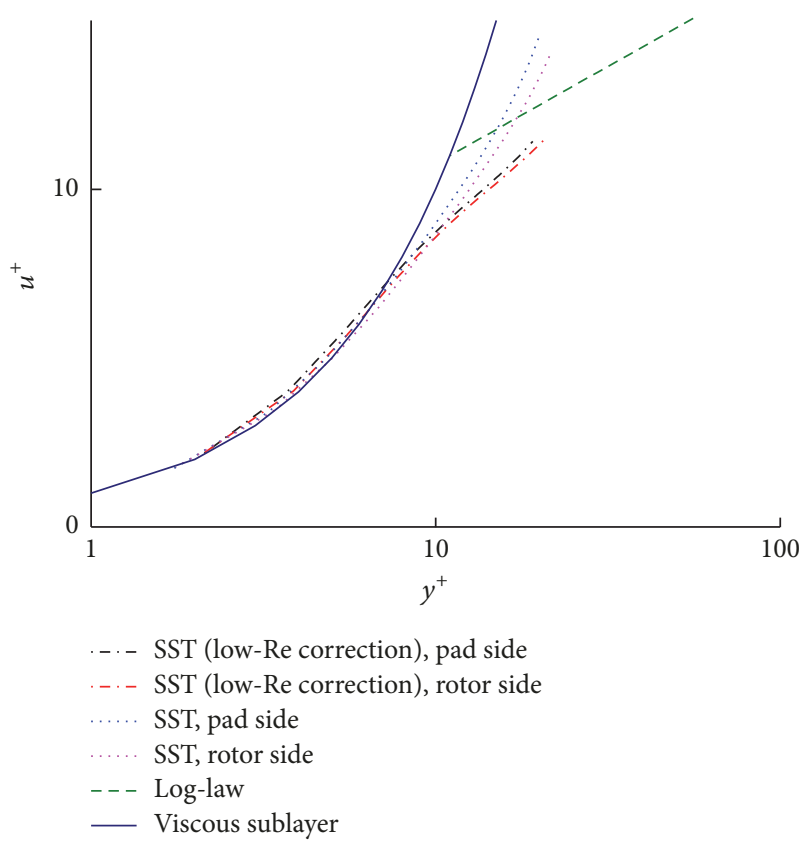

Figure $14: u^{+}$and $y^{+}$in $40^{\circ}$ from the inlet.

\section{Conclusions}

A three-dimensional model with a structured mesh for a tilting-pad journal bearing is established in this study. A total of fourteen turbulence models and laminar model are utilized to simulate the superlaminar oil flow at $3000 \mathrm{r} / \mathrm{min}$ and under $180 \mathrm{kN}$ loading. The simulation results are then compared with the experimental data obtained by Taniguchi et al. [25]. The conclusions of the current study are summarized as follows:

(i) Compared with experimental data, the laminar and classical turbulence models cannot accurately simulate the temperature and pressure distributions. Therefore, the laminar and classical fully developed turbulent models fail to adapt to superlaminar flow.

(ii) The transition turbulence models simulate the rapidly decreasing turbulent viscosity ratio and consider the oil flow around the outlet as laminar. This pattern is different from the change tendency of the experimental Reynolds number. Thus, the transition models generally obtain similar laminar simulation results and cannot properly simulate oil flow properly with an evident difference between the simulated THD characteristics and experimental results.

(iii) The performances of the low-Re correction in different turbulence models vary. The $k-\varepsilon$ models with lowRe correction simulate the oil flow as laminar, which results in unacceptable differences in the temperature and pressure results and experimental data. However, the results of the SST model with low-Re correction are consistent with the experimental data. The low-Re correction for SST is the best correction to simulate the superlaminar oil flow in the tilting-pad journal bearing among the compared models.

(iv) The velocity profiles indicate that the buffer layer plays the most important role for the turbulence boundary layer of the bearing. This finding suggests that the superlaminar flow mechanism is near-wall turbulence. Although the classical fully developed turbulence models cannot accurately predict the buffer layer, an appropriate low-Re correction can address this problem and help the SST model with low-Re correction yield suitable results for the superlaminar flow in the bearing.

In summary, this study reveals the physical meaning of the superlaminar oil flow in bearings and the significant importance of the buffer layer in the superlaminar flow. A suitable turbulence model, the SST model with low-Re correction, is pointed out for simulating the superlaminar flow accurately.

\section{Nomenclature}

$\begin{array}{ll}C_{\mu}: & \text { Constant } \\ C_{1 \varepsilon}: & \text { Constant } \\ C_{2 \varepsilon}: & \text { Constant } \\ C_{2}: & \text { Constant } \\ d: & \text { Film thickness } \\ f: & \text { Body force } \\ i, j, l: & \text { Three dimensions } \\ k: & \text { Turbulence kinetic energy } \\ P: & \text { Stress tensor } \\ R: & \text { Radius of the rotor } \\ \operatorname{Re}^{*} & \text { Reynolds number } \\ \operatorname{Re}^{*}: & \text { Reduced Reynolds number } \\ \mathrm{Re}_{t}: & \text { Turbulent Reynolds number } \\ t: & \text { Time } \\ u: & \text { Flow velocity } \\ u^{+}: & \text {Dimensionless velocity } \\ u_{\tau}: & \text { Friction velocity } \\ y: & \text { Distance to the nearest wall } \\ y^{+}: & \text {Wall coordinate } \\ \alpha *: & \text { Turbulent viscosity coefficient } \\ \varepsilon: & \text { Turbulent dissipation rate } \\ \mu: & \text { Kinetic viscosity } \\ \rho: & \text { Density } \\ \sigma_{k}: & \text { Constant } \\ \sigma_{\varepsilon}: & \text { Constant } \\ \tau_{w}: & \text { Wall shear stress } \\ \omega: & \text { Specific dissipation rate. } \\ & \end{array}$

\section{Conflicts of Interest}

The authors declare that they have no conflicts of interest.

\section{References}

[1] J. C. Fisher, "The fracture of liquids," Journal of Applied Physics, vol. 19, no. 11, pp. 1062-1067, 1948. 
[2] H. Temperley, “Two-dimensional ising models," in Phase Transitions and Critical Phenomena, C. Domb and M. S. Green, Eds., Academic Press, Cambridge, Mass, USA, 1974.

[3] L. Floberg, "Lund Technical University," Journal of Tribology, vol. 6, no. 4, pp. 145-146, 1973.

[4] D. Dowson and C. M. Taylor, "Cavitation in bearings", Annual Review of Fluid Mechanics, vol. 11, pp. 35-66, 1979.

[5] C. M. Woods and D. E. Brewe, "Solution of the Elrod algorithm for a dynamically loaded journal bearing using multigrid techniques," Journal of Tribology, vol. 111, no. 2, pp. 302-308, 1989.

[6] D. E. Brewe and B. O. Jacobson, "The effect of vibration amplitude on vapour cavitation in journal bearings," Wear, vol. 115, no. 1-2, pp. 63-73, 1987.

[7] X.-S. Li, Y. Song, Z.-R. Hao, and C.-W. Gu, "Cavitation mechanism of oil-film bearing and development of a new gaseous cavitation model based on air solubility," Journal of Tribology, vol. 134, no. 3, Article ID 031701, 2012.

[8] X. Li, Y. Song, Z. Hao, and C. Gu, "Cavitation Mechanism of OilFilm Bearing and Development of a New Gaseous Cavitation Model Based on Air Solubility," Journal of Tribology, vol. 134, no. 3, pp. 1047-1052, 2012.

[9] Z.-R. Hao and C. W. Gu, "Numerical modeling for gaseous cavitation of oil film and non-equilibrium dissolution effects in thrust bearings," Tribology International, vol. 78, pp. 14-26, 2014.

[10] Y. Song, C.-W. Gu, and X. Ren, "Development and validation of a gaseous cavitation model for hydrodynamic lubrication," Proceedings of the Institution of Mechanical Engineers, Part J: Journal of Engineering Tribology, vol. 229, no. 10, pp. 1227-1238, 2015.

[11] Y. Song, X. Ren, C.-W. Gu, and X.-S. Li, "Experimental and numerical studies of cavitation effects in a tapered land thrust bearing," Journal of Tribology, vol. 137, no. 1, Article ID 011701, 2015.

[12] D. G. Huang and Y. Q. Zhuang, "Temperature and cavitation," Proceedings of the Institution of Mechanical Engineers, Part C: Journal of Mechanical Engineering Science, vol. 222, no. 2, pp. 207-211, 2008.

[13] D. Huang, Y. Zhuang, and R. Cai, "A computational method for cavitational flows based on energy conservation," Proceedings of the Institution of Mechanical Engineers, Part C: Journal of Mechanical Engineering Science, vol. 221, no. 11, pp. 1333-1338, 2007.

[14] Y. Y. Zhang, X. J. Sun, and D. G. Huang, "A numerical study on cavitation suppression using local cooling," International Journal of Fluid Machinery \&amp; Systems, vol. 3, no. 4, pp. 292300,2010 .

[15] Y. Wang, X. J. Sun, Y. J. Dai, G. Q. Wu, Y. Cao, and D. G. Huang, "Numerical investigation of drag reduction by heat-enhanced cavitation," Applied Thermal Engineering, vol. 75, pp. 193-202, 2015.

[16] D. F. Wilcock, "Super Laminar Flow in Bearings: A Review of the Second Leeds-Lyon Symposium, Lyons, France, September 17-19, 1975," Journal of Lubrication Technology, vol. 98, no. 1, pp. $5-7,1976$.

[17] D. F. Wilcock, "Turbulence in High Speed Journal Bearing," Trans. ASME, vol. 173, no. 1, pp. 825-834, 1950.

[18] V. N. Constantinescu, "On turbulent lubrication," Archive Proceedings of the Institution of Mechanical Engineers, vol. 173, pp. 881-900, 1959.
[19] C. W. Ng and C. H. T. Pan, "A linearized turbulent lubrication theory," Journal of Fluids Engineering, vol. 87, no. 3, pp. 675-682, 1965.

[20] G. G. Hirs, "A bulk-flow theory for turbulence in lubricant films," Journal of Lubrication Technology, vol. 95, no. 2, pp. 137146, 1973.

[21] T. Suganami and A. Z. Szeri, "Parametric study of journal bearing performance: the $80 \mathrm{deg}$ partial arc bearing," Journal of Lubrication Technology, vol. 101, no. 4, pp. 486-491, 1979.

[22] S. J. Chowdhury and G. Ahmadi, "Thermohydrodynamic analysis of wide thrust bearings operating in turbulent inertial flow regimes," Journal of Tribology, vol. 110, no. 2, pp. 327-334, 1988.

[23] D. Ives and W. B. Rowe, "The performance of hybrid journal bearings in the superlaminar flow regimes," Tribology Transactions, vol. 35, no. 4, pp. 627-634, 1992.

[24] H. Hashimoto, "Surface roughness effects in high-speed hydrodynamic journal bearings," Journal of Tribology, vol. 119, no. 4, pp. 776-780, 1997.

[25] S. Taniguchi, T. Makino, K. Takeshita, and T. Ichimura, “Thermohydrodynamic analysis of large tilting-pad journal bearing in laminar and turbulent flow regimes with mixing," Journal of Tribology, vol. 112, no. 3, pp. 542-550, 1990.

[26] B. Maneshian and S. A. Gandjalikhan Nassab, “Thermohydrodynamic characteristics of journal bearings running under turbulent condition," International Journal of Engineering, Transactions A: Basics, vol. 22, no. 2, pp. 181-194, 2009.

[27] A. A. Solghar and S. A. Gandjalikhan Nassab, "Numerical analysis of turbulent lubrication in plain full journal bearings," Industrial Lubrication and Tribology, vol. 65, no. 2, pp. 91-99, 2013.

[28] L. Bouard, M. Fillon, and J. Frêne, "Comparison between three turbulent models - Application to thermohydrodynamic performances of tilting-pad journal bearings," Tribology International, vol. 29, no. 1, pp. 11-18, 1996.

[29] A. Ding and X. Ren, "Validation of Turbulence Models for the Superlaminar Flows in Journal Bearings," in Proceedings of the ASME Turbo Expo 2017: Turbomachinery Technical Conference and Exposition, Charlotte, NC, USA.

[30] T. Dimond, A. Younan, and P. Allaire, "A review of tilting pad bearing theory," International Journal of Rotating Machinery, vol. 2011, Article ID 908469, 23 pages, 2011.

[31] H. Schlichting, Boundary-Layer Theory, 2000. 


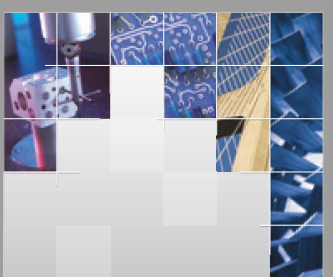

\section{Enfincering}
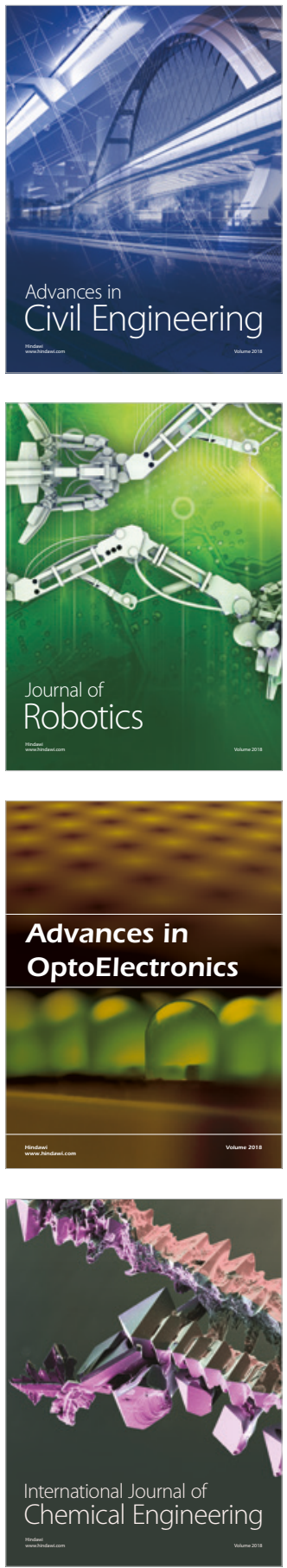

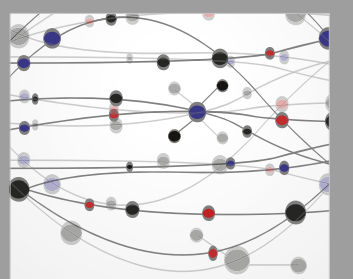

\section{Rotating \\ Machinery}

The Scientific World Journal

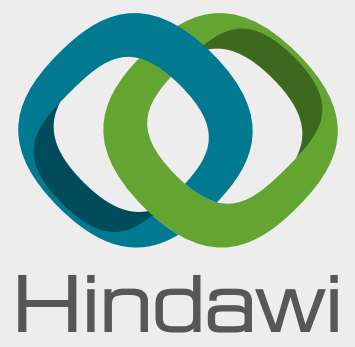

Submit your manuscripts at

www.hindawi.com
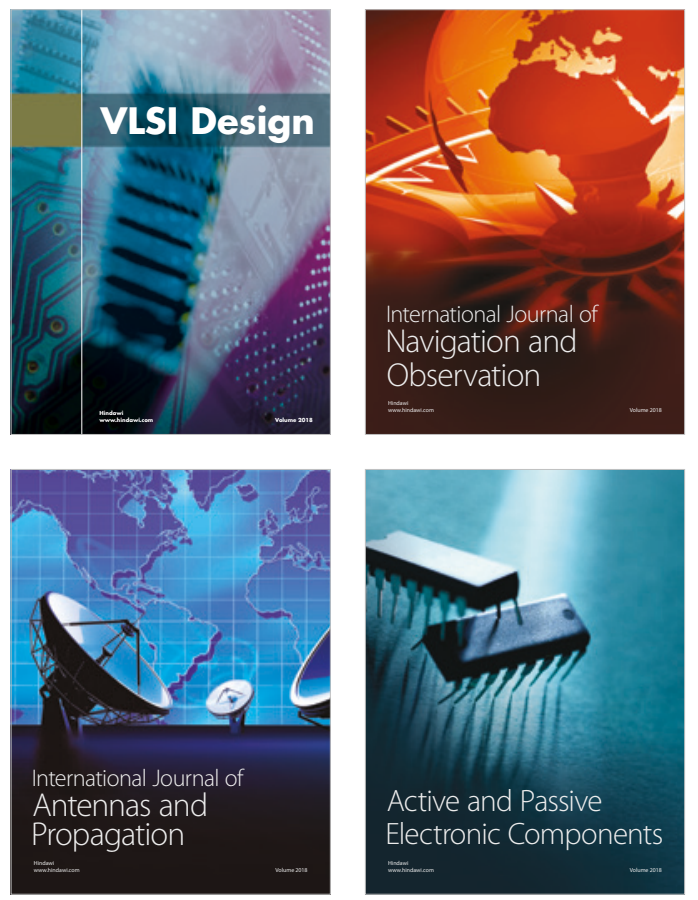
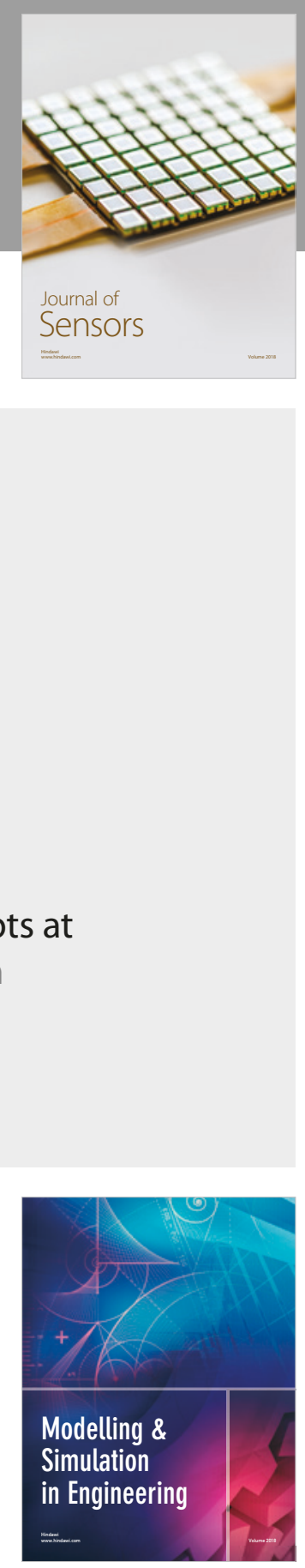

\section{Advances \\ Multimedia}
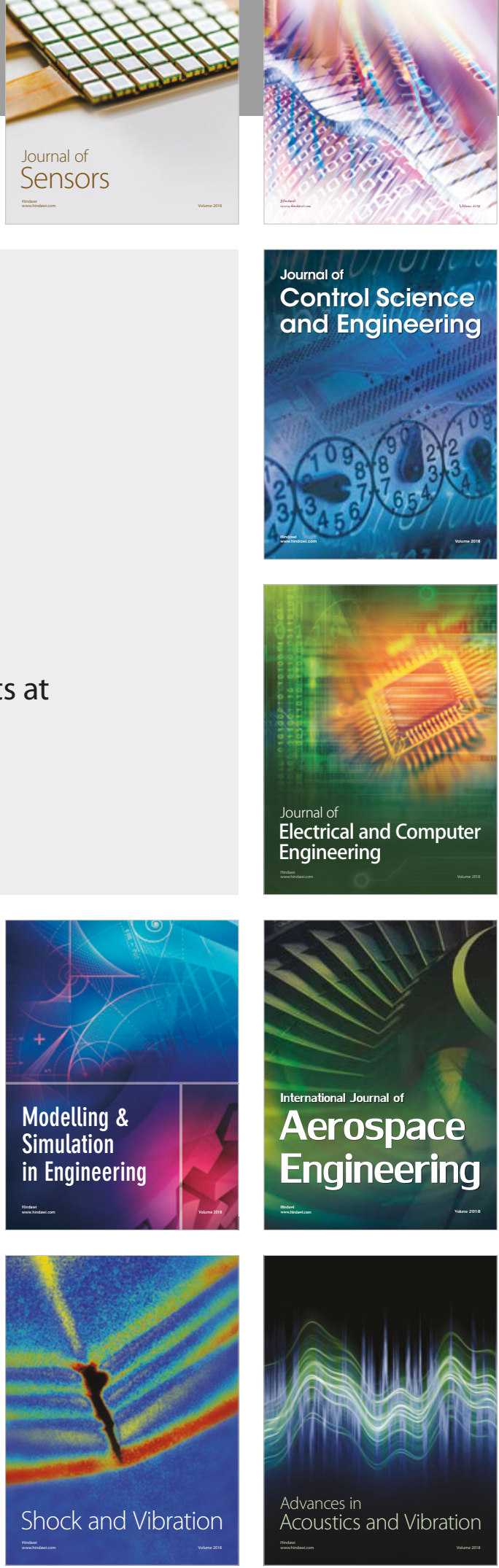